\title{
Binary Stars in the Orion Nebula Cluster
}

\author{
Rainer Köhler ${ }^{1} \dagger$ Monika G. Petr-Gotzens ${ }^{2}$, Mark J. McCaughrean ${ }^{3}$, \\ Jerome Bouvier $^{4}$, Gaspard Duchêne ${ }^{4}$, Andreas Quirrenbach ${ }^{5}$ \\ and Hans Zinnecker ${ }^{6}$ \\ ${ }^{1}$ Sterrewacht Leiden, P.O. Box 9513, NL-2300 RA Leiden, The Netherlands \\ email: koehler@strw.leidenuniv.nl \\ ${ }^{2}$ European Southern Observatory, Karl-Schwarzschild-Str. 2, 85748 Garching bei München, \\ Germany \\ ${ }^{3}$ School of Physics, University of Exeter, Stocker Road, Exeter EX4 4QL, Devon, UK \\ ${ }^{4}$ Laboratoire d'Astrophysique de Grenoble, Université Joseph Fourier, \\ BP 53, 38041 Grenoble Cedex 9, France \\ ${ }^{5}$ ZAH Landessternwarte, Königstuhl, Heidelberg, Germany \\ ${ }^{6}$ Astrophysikalisches Institut Potsdam, An der Sternwarte 16, 14482 Potsdam, Germany
}

\begin{abstract}
We report on a high-spatial-resolution survey for binary stars in the periphery of the Orion Nebula Cluster, at 5-15 arcmin $(0.65-2$ pc) from the cluster center. We observed 228 stars with adaptive optics systems, in order to find companions at separations of $0.13-1.12$ arcsec (60 - $500 \mathrm{AU})$, and detected 13 new binaries. Combined with the results of Petr (1998), we have a sample of 275 objects, about half of which have masses from the literature and high probabilities to be cluster members. We used an improved method to derive the completeness limits of the observations, which takes into account the elongated point spread function of stars at relatively large distances from the adaptive optics guide star. The multiplicity of stars with masses $>2 \mathrm{M}_{\odot}$ is found to be significantly larger than that of low-mass stars. The companion star frequency of low-mass stars is comparable to that of main-sequence M-dwarfs, less than half that of solar-type main-sequence stars, and 3.5 to 5 times lower than in the Taurus-Auriga and Scorpius-Centaurus star-forming regions. We find the binary frequency of low-mass stars in the periphery of the cluster to be the same or only slightly higher than for stars in the cluster core $\left(<3^{\prime}\right.$ from $\theta^{1} \mathrm{C}$ Ori $)$. This is in contrast to the prediction of the theory that the low binary frequency in the cluster is caused by the disruption of binaries due to dynamical interactions. There are two ways out of this dilemma: Either the initial binary frequency in the Orion Nebula Cluster was lower than in Taurus-Auriga, or the Orion Nebula Cluster was originally much denser and dynamically more active. A detailed report of this work has been published in Astronomy $\mathcal{E}$ Astrophysics (Köhler et al. 2006).
\end{abstract}

Keywords. techniques: high angular resolution, binaries: close, stars: formation, stars: premain-sequence open clusters and associations: individual (Orion Nebula Cluster)

\section{Introduction}

Stellar multiplicity is very high among young low-mass stars, with companion star frequencies close to $100 \%$ for young stars in well-known nearby star-forming T associations like Taurus-Auriga (Leinert et al. 1993, Ghez et al. 1993, Ghez et al. 1997, Duchêne 1999). On the other hand, high binary frequencies are not observed among low-mass stars in stellar clusters like the Orion Nebula Cluster (e.g., Prosser et al. 1994, Padgett et al. 1997, Petr et al. 1998, Petr 1998, Simon et al. 1999, Scally et al. 1999, McCaughrean 2001). The reason for this discrepancy is still unclear. Theoretical explanations include:

$\dagger$ Present address: ZAH Landessternwarte, Königstuhl, 69117 Heidelberg, Germany. 


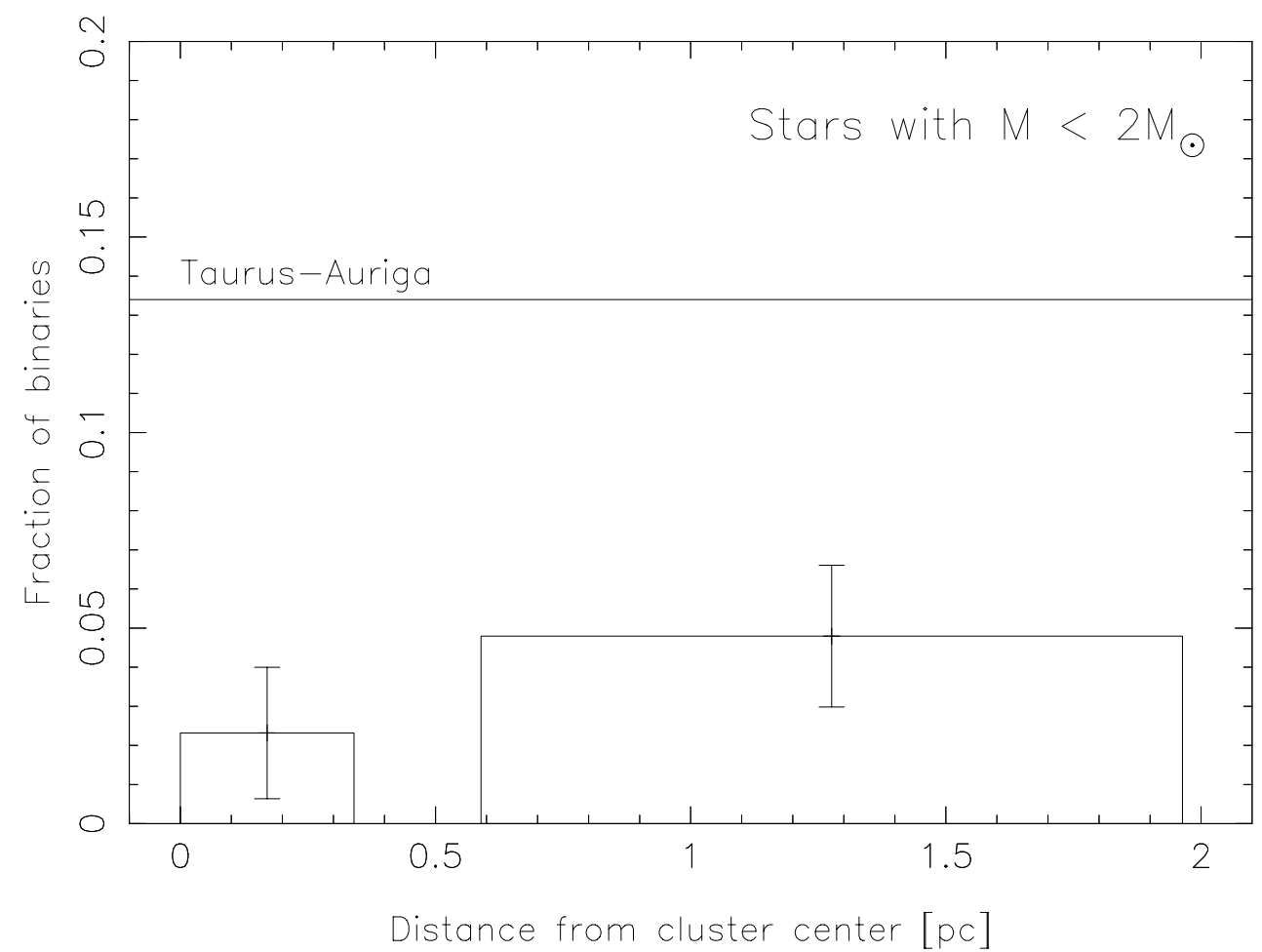

Figure 1. The binary frequency of low-mass stars $\left(M<2 \mathrm{M}_{\odot}\right)$ in the center and the periphery of the Orion Nebula Cluster.

- Binaries are destroyed in close encounters with other stars (e.g., Kroupa 1995, Kroupa et al. 1999)

- The formation rate of binaries depends on the precollapse cloud conditions (e.g., temperature) (Durisen \& Sterzik 1994, Sterzik et al. 2003)

The interaction time scale depends on the stellar density, so less binaries should be destroyed in the outer parts of the cluster.

\section{Observations and Results}

Our target list comprises some 230 stars in 52 fields, located at $5^{\prime}-15^{\prime}(0.7-2 \mathrm{pc})$ from the cluster center. The adaptive optics systems at the 3.6-m telescope on La Silla and the Keck Telescope on Mauna Kea were used to observe them in the $K$-band. We found 13 companions in the separation range $0^{\prime \prime} .13-1^{\prime \prime} .12$ (60 - $\left.500 \mathrm{AU}\right)$. For comparison, we use the sample by Petr (1998), which contains 114 stars in the cluster core $\left(<3^{\prime}\right.$ or $0.4 \mathrm{pc}$ from the center). We find the binary frequency of low-mass stars in the periphery of the cluster to be only slightly higher than in the core. In particular, the binary frequency in the periphery is significantly lower than in Taurus-Auriga.

\section{Conclusions}

We find no statistically significant difference of the binary frequency of low-mass stars between core and periphery. These results do not support the hypothesis that the binary frequency in Orion was initially as high as in Taurus and later reduced by dynamical interactions, unless the Orion Nebula Cluster was much denser in the past. 


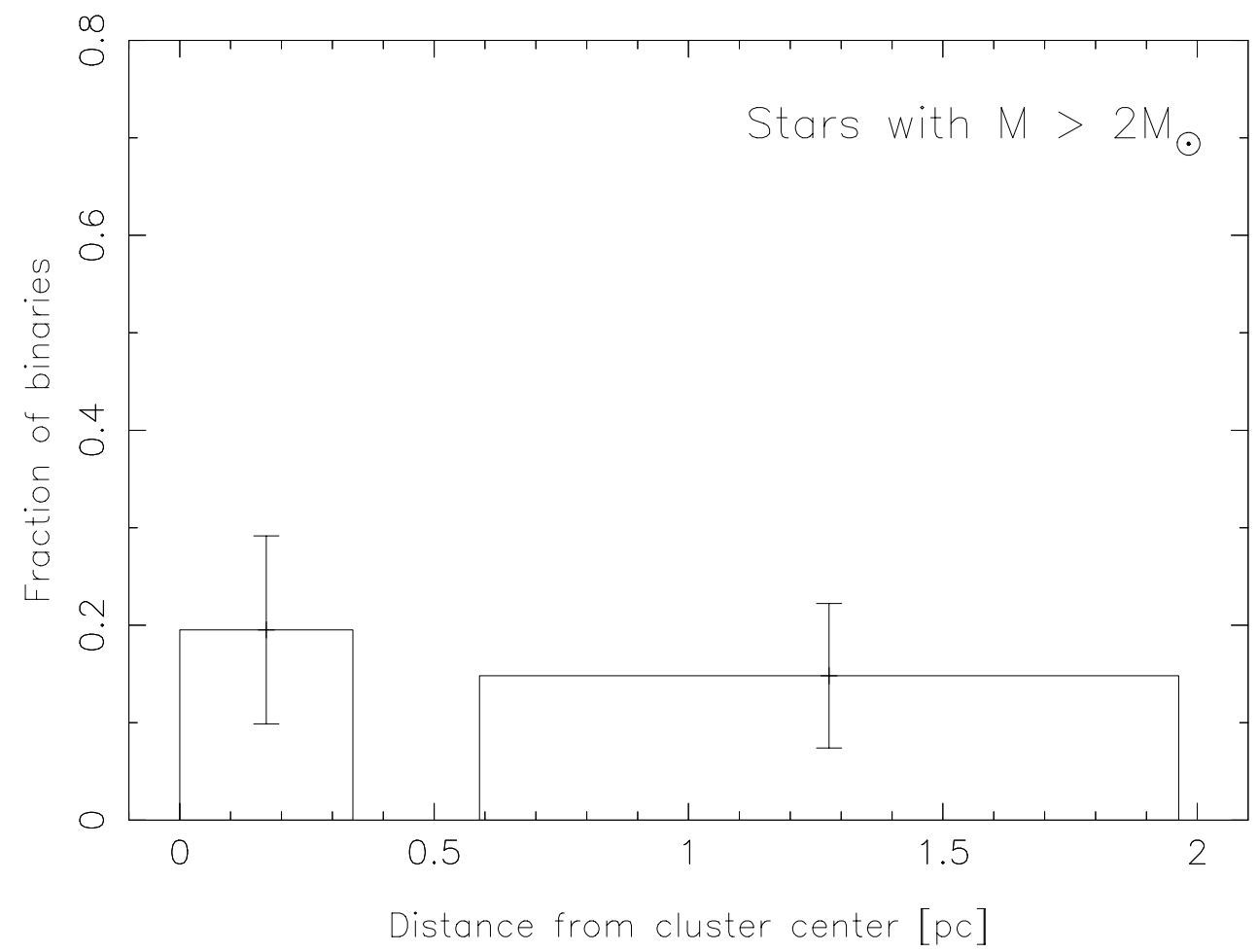

Figure 2. The binary frequency of intermediate- to high-mass stars $\left(M>2 M_{\odot}\right)$ in the center and the periphery of the Orion Nebula Cluster.

\section{Acknowledgements}

We thank the organizers for the opportunity to present our work.

\section{References}

Duchêne, G. 1999, A\&GA 341, 547

Durisen, R.H. \& Sterzik, M.F. 1994, A\&A 286, 84

Ghez, A.M., Neugebauer, G., \& Matthews, K. 1993, AJ 106, 2005

Ghez, A.M., McCarthy, D.W., Patience, J., \& Beck, T. 1997, AJ 481, 378

Köhler, R., Petr-Gotzens, M.G., McCaughrean, M.J., Bouvier, J., Duchêne, G., Quirrenbach, A., \& Zinnecker, H. 2006, A\&̈A 458, 461

Kroupa, P. 1995, MNRAS 277, 1491

Kroupa, P., Petr, M., \& McCaughrean, M. 1999, New Astronomy 4, 495

Leinert, Ch., Zinnecker, H., Weitzel, N., et al. 1993, A\&A 278, 129

McCaughrean, M.J. 2001, in: The Formation of Binary Stars, proceedings of IAU Symp. No. 200, eds. H. Zinnecker and R.D. Mathieu, ASP Conference Series, p. 169

Padgett, D.L., Strom, S.E., \& Ghez, A. 1997, ApJ 477, 705

Petr, M.G., Coudé du Foresto, V., Beckwith, S.V.W., Richichi, A., \& McCaughrean, M.J. 1998, ApJ 500, 825

Petr, M.G. 1998, PhD Thesis, University of Heidelberg

Prosser, C.F., Stauffer, J.R., Hartmann, L., et al. 1994, ApJ 421, 517

Scally, A., Clarke, C., \& McCaughrean M.J. 1999, MNRAS 306, 253

Simon, M., Close, L.M., \& Beck, T.L. 1999, AJ 117, 1375

Sterzik, M.F., Durisen, R.H., \& Zinnecker, H. 2003, A\&A 411, 91 